\title{
Drag and Heat Transfer of Metal and Oxide Agglomerates in Flow of Combustion Products of Solid Propellant
}

\author{
V. Emel'yanov, I. Teterina, K. Volkov \\ Baltic State Technical University, St Petersburg, 190005, Russia \\ dsci@mail.ru
}

\begin{abstract}
Development of tools for modeling the motion of agglomerated particles with a complex composition and shape in a flow of combustion products is of interest for problems related to the description of the motion of the condensed phase in the internal channels of solid rocket motors. An approach is developed to simulate unsteady processes that occur when a viscous incompressible fluid flows around a particle consisting of an aluminum droplet and a condensed oxide particle attached to it. Methods for taking into account the sliding of the attached oxide particle over the surface of the aluminum droplet are proposed. The computational results are used to find the drag and heat transfer coefficients of a non-spherical particle at flow velocities corresponding to the formation of separation zones. The developed model is one of the components of a more general mathematical model designed for calculating two-phase flows of combustion products and created with a multi-scale approach to simulation of two-phase flows.
\end{abstract}

Keywords: computational fluid dynamics, solid rocket nozzle, agglomeration, droplet.



Geometrical model of a droplet with oxide cap

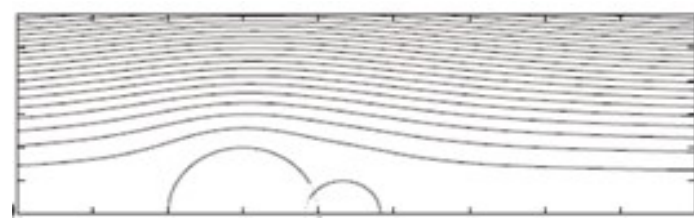

Streamlines of inviscid flow around a droplet



Geometry of a droplet

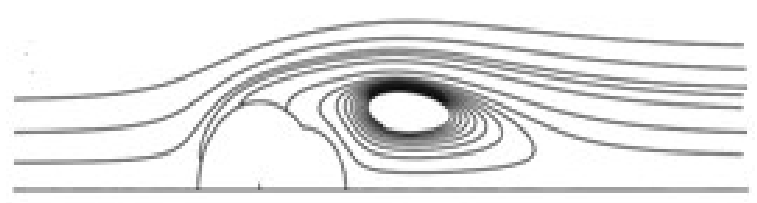

Streamlines of viscous flow around a droplet at $\mathrm{Re}=100$ 


\title{
Сопротивление и теплообмен металл- оксидных агломератов в потоке продуктов сгорания твердого топлива
}

\author{
К.Н. Волков, В.Н. Емельянов, И.В. Тетерина \\ Балтийский государственный технический университет, \\ Россия, Санкт-Петербург, 190005, 1-я Красноармейская ул., 1 \\ dsci@mail.ru
}

\begin{abstract}
Аннотация
Создание средств моделирования движения частиц-агломератов, имеющих сложный состав и форму, в потоке продуктов сгорания представляет интерес для задач, связанных с описанием движения конденсированной фазы во внутренних каналах ракетных двигателей твердого топлива. Разрабатывается подход, предназначенный для моделирования нестационарных процессов, реализующихся при обтекании частицы, состоящей из капли алюминия и присоединенной к ней частицы конденсированного оксида, потоком вязкой несжимаемой жидкости. Предлагаются методы учета скольжения присоединенной частицы по поверхности основной частицы. Результаты расчетов используются для нахождения коэффициентов сопротивления и теплоотдачи частицы несферической формы при скоростях обтекания, соответствующих образованию отрывных зон. Построенная модель является одной из составных частей более общей математической модели, предназначенной для расчета двухфазных течений продуктов сгорания и построенной на основе многомасштабного подхода к расчету двухфазных течений.
\end{abstract}

Ключевые слова: вычислительная газовая динамика, двигатель, агломерация, капля.

\section{1. Введение}

Металлические добавки в виде высокодисперсного порошка (в основном, алюминия) входят в состав многих типов современных смесевых твердых ракетных топлив (СТРТ). Они призваны обеспечить достижение требуемого уровня энергетических характеристик и демпфирование неуправляемых акустических колебаний параметров рабочего тела в камерах сгорания крупногабаритных ракетных двигателей на твердом топливе (РДТТ). Включение в состав СТРТ добавок алюминия (массовая концентрация металла достигает $20 \%$ ) существенно усложняет механизм их горения и приводит к возникновению ряда проблем при организации работы двигателя (двухфазные потери удельного импульса тяги, выпадение частиц конденсированной фазы на элементы конструкции, эрозионное воздействие конденсированных продуктов сгорания на теплозащитные покрытия). В тоже время, частицы, образующиеся при сгорании топлива, представляют собой основной источник потерь акустической энергии, которая поступает из зоны горения топлива [1], что стабилизирует горение СТРТ, которое в другом случае оказывается нестабильным. Совокупность процессов, происходящих с конденсированной фазой в рабочем пространстве камеры сгорания, приводит к изменению характеристик двухфазного потока продуктов сгорания и играет важную роль в формировании спектра размеров частиц на входе в сопло.

За время пребывания в камере сгорания двигательной установки частицы алюминия претерпевают ряд физико-химических превращений, начинающихся в поверхностном слое топлива и приводящих к формированию двухфазного потока продуктов сгорания. Одной из 
особенностей горения твердых топлив с добавками алюминия является слияние (агломерация) расплавленных частиц металла и его оксида в поверхностном слое горящего топлива в капли, размер которых на порядок превышает размеры исходных частиц металла.

В газовую фазу конденсированные продукты сгорания алюминия поставляются в виде частиц высокодисперсного оксида субмикронного размера (размер таких частиц изменяется от 10 нм до 20 мкм, а среднемассовый размер частиц окисла металла находится в интервале $r_{43}=0.25 \div 2.5$ мкм) и частиц-агломератов, размер которых достигает десятков и сотен микрон [2]. Концентрация частиц окиси и частиц-агломератов у поверхности горящего топлива определяется свойствами топлива, в частности, дисперсностью и количеством окислителя. По своему строению частицы агломераты представляют собой совокупность капель $\mathrm{Al}_{\text {и }} \mathrm{Al}_{2} \mathrm{O}_{3}$, в которых имеют место газовые включения. Свойства таких образований близки к равновесным, при которых поверхностная энергия стремится к минимальному значению. В зависимости от особенностей внутреннего строения агломераты разделяют на два типа [3]. К первому типу относятся так называемые матричные агломераты, состоящие из частиц $\mathrm{Al}_{2} \mathrm{O}_{3}$ сферической формы, в которые внедрены отдельные частицы Al. Агломераты второго типа представляют собой частицы металла, на поверхности которых в том или ином количестве в виде капли находится окись, называемая нашлепкой окиси (oxide cap) $[4,5]$ (рис. 1).

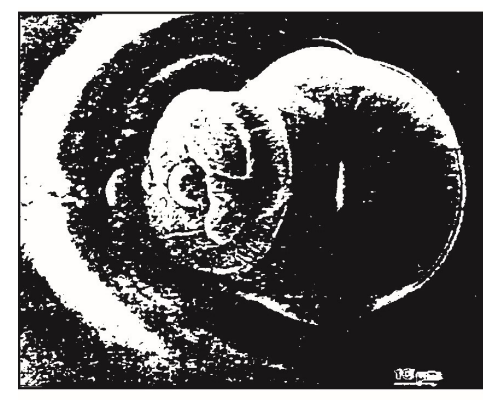

a)

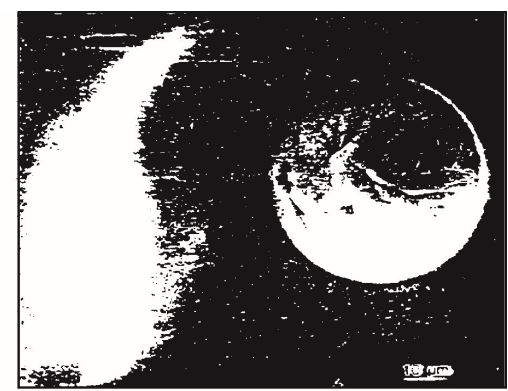

б)

Рис. 1. Фотография частицы алюминия диаметром 70 мкм спустя 1.5 мкс $(a)$ и 5.5 мкс (б ) после воспламенения [5]

Течения двухфазных смесей, состоящих из вязкой жидкости и взвешенных в ней твердых частиц или капель, представляют собой сложную систему, поведение которой определяется взаимосвязью и взаимовлиянием многих факторов и физических процессов. На границе частицы, находящейся в контакте с внешней подвижной средой, возникает система сил и моментов взаимодействия. Изучение свойств этих сил и моментов, их зависимости от закона движения частицы и ее геометрических характеристик имеет большое практическое значение. Нестационарные эффекты, проявляющиеся в силовом воздействии несущего потока на частицу, в существенной степени изменяют характер движения частицы и интенсифицируют процессы тепломассопереноса в двухфазном течении [6].

При низких числах Рейнольдса, соответствующих стоксовому режиму течения около частиц различной формы (сфера, сплюснутый и втянутый эллипсоиды вращения, диск, стержень), для нахождения распределений характеристик потока и силы сопротивления применяются теоретические методы. При малых числах Рейнольдса конвективными слагаемыми в уравнениях Навье-Стокса пренебрегается. Функция тока вводится таким образом, чтобы удовлетворить уравнению неразрывности. Уравнения Стокса преобразуются к одному уравнению для функции тока, которое решается при помощи метода разделения переменных с граничными условиями однородности потока вдали от частицы и условиями непротекания и прилипания на ее поверхности.

Геометрическое моделирование конгломератов сложной формы рассматривается в работах $[7,8]$. Расчет сопротивления частиц несферической формы и системы из двух пересекающихся сфер в стоксовом режиме течения обсуждается в работах $[9,10]$. В работах $[11,12]$ 
рассматривается сопротивление объекта, состоящего из двух сфер одинакового диаметра, центры которых располагаются на фиксированном расстоянии друг от друга, а гидродинамическое взаимодействие между сферами одинакового диаметра, составляющими различные конфигурации, обсуждается в работах $[13,14]$ в зависимости от числа Рейнольдса и расстояния между частицами. Исследования проводятся для двух и трех сфер, центры которых располагаются на одной линии, а также для шести и восьми сфер, расположенных в вершинах шестиугольника и вершинах куба (основная частица находится в центре такой конфигурации). Стоксовое обтекание конгломерата, состоящего из сфер одинакового диаметра, моделируется в работе [15].

Точное решение задачи об обтекании и вращении системы из двух соприкасающихся сфер произвольного радиуса (sphere doublet) сдвиговым потоком дается в работе [16] (рассматривается стоксовый режим течения, когда число Рейнольдса, рассчитанное по сдвигу скорости, является малым). В работе [17] приводятся данные численного моделирования по обтеканию и сопротивлению двух соприкасающихся сфер ламинарным и турбулентным потоком. Для расчетов применяются уравнения Навье - Стокса и метод решеточных уравнений Больцмана (Lattice Boltzmann Method). Влияние стенки на сопротивление системы, состоящей из случайно расположенных сфер одинакового диаметра, рассматривается в работе [18]. Сопротивление твердой сферы в жидкой оболочке рассчитывается в работе [19] в рамках приближения малых чисел Рейнольдса.

Обтекание двух пересекающихся кругов (double circle) или сфер (double sphere) течением невязкой и вязкой несжимаемой жидкости в стоксовом режиме и их сопротивление обсуждаются в работе [20] (материалы двух тел считаются одинаковыми). Круги или сферы пересекаются под углом $\pi / n$, где $n$ - целое число. Для нахождения поля течения в плоском случае при обтекании двойного круга или сферы невязкой жидкостью применяется теория функций комплексной переменной (вводится комплексный потенциал, который удовлетворяет уравнению Лапласа). В осесимметричном случае вводится функция тока, которая удовлетворяет бигармоническому уравнению. В вязкой жидкости полагается, что касательные напряжения на поверхности круга или сферы равняются нулю. При произвольном угле пересечения сфер для нахождения поля течения около двойной сферы и ее сопротивления применяются тороидальные и бицилиндрические координаты, что позволяет получить решение задачи в квадратурах через конические функции Мехлера (Mehler conical function) [21, 22]. Полученные результаты находят применение при разработке соответствующих вычислительных алгоритмов [23].

Формирование агломератов при горении твердого топлива рассматривается в работах $[24,25]$. Модели основаны на экспериментальных данных, полученных при изучении эволюции агломератов в составе потока продуктов сгорания. Разработанные модели являются составными частями комплексного описания эволюции конденсированных продуктов в камере сгорания РДТТ. Математическая модель горения частиц, состоящих из алюминия и его оксида, в двухфазном потоке построена в работе [26]. Наличие частицы оксида нарушает сферическую симметрию, допущение о которой используется во многих моделях. Учет обдувающего потока производится в рамках модели приведенной пленки [27]. В работе [28] для описания процессов около сферической частицы, обдуваемой высокотемпературным потоком газа, используются нестационарные уравнения Навье - Стокса в осесимметричной постановке. Их решение получено для чисел Рейнольдса порядка 100 при значительной разности температур набегающего потока и поверхности сферы. Возникновение зоны интенсивной химической реакции за кормой сферической частицы вызывает волну давления, прохождение которой через частицу приводит к значительным колебаниям коэффициента сопротивления и, в меньшей степени, коэффициента теплоотдачи. Численное моделирование двухфазных турбулентных течений в каналах заряда твердого топлива и влияния частиц конденсированной фазы на демпфирование колебаний давления в камере сгорания проводится в работе [29]. 
Для нахождения коэффициента сопротивления агломератов алюминия применяются соотношения, полученные для твердой сферы. Обзор экспериментальных и численных результатов по горению агломератов дается в работе [30], в которой также обсуждаются различные корреляционные зависимости для нахождения времени горения.

При горении составных агломератов наличие частицы окиси, покрывающей каплю алюминия, нарушает сферическую симметрию процесса. Содержание оксида алюминия в агломерате может составлять более 50 \%. Частицы высокодисперсного оксида осаждаются на частицах-агломератах, увеличивая массу оксида в их составе. При определенных условиях имеет место химическое взаимодействие между конденсированными включениями металла и его оксида в агломератах с образованием газообразных продуктов, что приводит к изменению структуры и раздуванию агломератов, выбросу газообразных продуктов и уносу оксида. Кинетика процесса взаимодействия алюминия и его оксида определяется температурой и структурой агломератов. Рост температуры агломерата, повышение температуры и окислительного потенциала газовой фазы способствуют интенсификации взаимодействия конденсированных капли алюминия и частицы оксида.

Учет взаимодействия металла и оксида агломератов имеет существенное значение для повышения точности прогнозирования параметров конденсированных продуктов сгорания. Несмотря на достаточно большой и разнообразный по содержанию материал, связанный с моделированием движения, эволюции и горения металл-оксидных агломератов в потоке продуктов сгорания, в литературе уделяется недостаточное внимание расчетам коэффициентов сопротивления и теплоотдачи частиц несферической формы. Имеющиеся данные относятся, в основном, к определению времени горения агломератов алюминия [31-34]. Коэффициенты сопротивления и теплоотдачи являются входными данными для моделирования двухфазных течений в каналах и соплах РДТТ при помощи эйлеровых или лагранжевых подходов. В расчетной практике при моделировании двухфазных потоков с несферическими частицами обычно используются коэффициенты сопротивления и теплоотдачи, полученные для сферы некоторого эквивалентного размера. Такой подход приводит к существенным погрешностям в определении траекторий частиц в канале заряда и времени выгорания частиц-агломератов $[35]$.

В данной работе проводится численное моделирование обтекания и вращения капли алюминия с присоединенной к ней частицей конденсированного оксида потоком вязкой несжимаемой жидкости (горение частицы не рассматривается). Результаты расчетов приводятся для различных чисел Рейнольдса и геометрических параметров, характеризующих форму составной частицы.

\section{2. Геометрия составной частицы}

Форма составной частицы зависит от содержания алюминия в частице $C_{1}$ и температуры. Температура частицы предполагается одинаковой по ее объему и равной температуре кипения $T_{b}$, которая определяется при помощи термодинамического расчета. Для описания геометрии частицы сложной формы, представляющей собой каплю металла с присоединенной окисной частицей, используется модель тела вращения, составленного из двух сфер разного диаметра.

Принимается, что частица образована двумя сферами с радиусами $R_{1}$ и $R_{2}$ (рис. 2). Их взаимное расположение зависит от краевого угла смачивания $\theta$. Угол смачивания определяется коэффициентами поверхностного натяжения на границах раздела $\mathrm{Al}-\mathrm{Al}_{2} \mathrm{O}_{3}, \mathrm{Al}$-газ, $\mathrm{Al}_{2} \mathrm{O}_{3}-$ газ и рассчитывается на основе уравнения (Young's equation)

$$
\cos \theta=\frac{\sigma_{s v}-\sigma_{s l}}{\sigma_{l v}}
$$


где $\sigma_{s v}, \sigma_{l v}$ и $\sigma_{s l}$ представляют собой коэффициенты поверхностного натяжения на границах раздела частица-газ $\left(\mathrm{Al}_{2} \mathrm{O}_{3}\right.$ и газ), капля-газ ( $\mathrm{Al}$ и газ) и частица-капля $\left(\mathrm{Al}_{2} \mathrm{O}_{3}\right.$ и $\left.\mathrm{Al}\right)$. Следует отметить, что, например, в работе [31] угол пересечения двух сфер фиксируется равным 90. Продуктами испарения с границы контакта капли и частицы оксида являются $\mathrm{Al}$ и $\mathrm{Al}_{2} \mathrm{O}$. На открытой поверхности алюминия идет гетерогенная реакция окисления. Образовавшийся оксид присоединяется к частице окиси. Продукты испарения из зоны контакта вдуваются в окружающий поток.

При известном угле смачивания форма частицы-агломерата определяется радиусом капли алюминия $R_{1}$, радиусом частицы присоединенного оксида $R_{2}$, радиусом круга, образованного пересечением двух сфер $R_{3}$, расстоянием между центрами сфер, представляющими собой каплю металла и частицу присоединенного оксида $H$, и двумя параметрами $H_{1}$ и $H_{2}$, которые имеют значения высот соответствующих сферических сегментов. Радиус круга, образованного пересечением двух сфер, находится из соотношения

$$
R_{3}=\frac{R_{1} R_{2}}{H} \sin \theta
$$

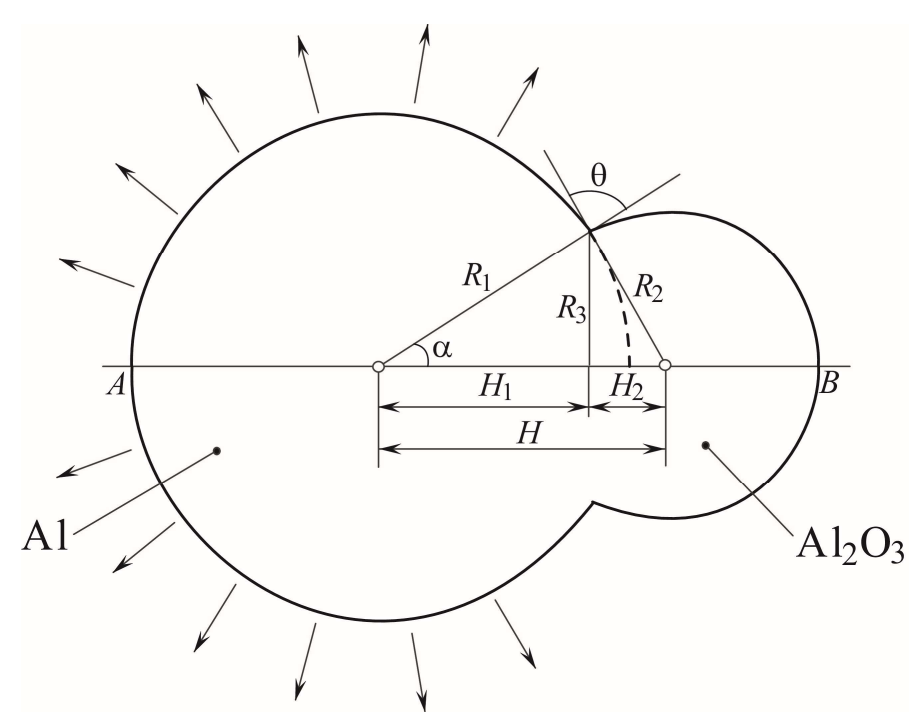

Рис. 2. Геометрия капли алюминия с присоединенной к ней частицей конденсированного оксида

Расстояние между центрами сфер, представляющими собой каплю металла и частицу присоединенного оксида, равняется

$$
H=R_{1}^{2}+R_{2}^{2}-2 R_{1} R_{2} \cos \theta
$$

Из рассмотрения треугольника со сторонами $R_{1}, R_{2}$ и $H$ следует, что

$$
R_{1}^{2}-H_{1}^{2}=R_{3}^{2}, R_{2}^{2}-H_{2}^{2}=R_{3}^{2}, H_{1}+H_{2}=H
$$

Линейный угол находится из соотношения

$$
\alpha=\arcsin \left(\frac{R_{3}}{R_{2}}\right)
$$

Телесный угол равняется

$$
\vartheta=2 \pi(1-\cos \alpha)
$$

Часть поверхности капли алюминия, покрытая частицей конденсированного оксида, рассчитывается по формуле 


$$
\beta=\frac{\vartheta}{2 \pi}=\frac{1}{2}(1-\cos \alpha)
$$

Масса частицы-агломерата равняется сумме масс капли алюминия и частицы присоединенного оксида

$$
m=m_{1}+m_{2}
$$

Эквивалентный радиус сферы такого же объема, что и частица-агломерат, определяется следующим образом

$$
R_{m}=\left[\frac{3}{4 \pi}\left(\frac{m_{1}}{\rho_{1}}+\frac{m_{2}}{\rho_{2}}\right)\right]^{1 / 3}
$$

Относительные массы алюминия и его оксида составляют

$$
C_{1}=\frac{m_{1}}{m_{1}+m_{2}}, C_{2}=\frac{m_{2}}{m_{1}+m_{2}},
$$

причем $C_{1}+C_{2}=1$. Средняя плотность частицы-агломерата находится из соотношения

$$
\rho=\left(\frac{C_{1}}{\rho_{1}}+\frac{1-C_{1}}{\rho_{2}}\right)^{-1}
$$

Массы капли-алюминия и частицы конденсированного оксида равняются $m_{1}=\rho_{1} V_{1}$ и $m_{2}=\rho_{2} V_{2}$. Объем капли алюминия рассчитывается как объем сферы радиусом $R_{1}$, что дает

$$
V_{1}=\frac{4}{3} \pi R_{1}^{3}
$$

Радиус капли алюминия составляет

$$
R_{1}=\left(\frac{3 m_{1}}{4 \pi \rho_{1}}\right)^{1 / 3}
$$

Объем частицы присоединенного оксида находится как разность между объемом сферического сегмента радиусом $R_{3}$ и высотой $R_{2}+H_{2}$ и объемом сферического сегмента радиусом $R_{3}$ и высотой $R_{1}-H_{1}$, что дает

$$
V_{2}=\frac{\pi}{6}\left(R_{2}+H_{2}\right)\left[3 R_{3}^{2}+\left(R_{2}+H_{2}\right)^{2}\right]-\frac{\pi}{6}\left(R_{1}-H_{1}\right)\left[3 R_{3}^{2}+\left(R_{1}+H_{1}\right)^{2}\right]
$$

Масса капли алюминия и масса частицы конденсированного оксида связаны при помощи соотношения

$$
8 \frac{1-C_{1}}{C_{1}} \frac{\rho_{1}}{\rho_{2}} R_{1}^{3}=\left(R_{2}+H_{2}\right)\left[3 R_{3}^{2}+\left(R_{2}+H_{2}\right)^{2}\right]-\frac{\pi}{6}\left(R_{1}-H_{1}\right)\left[3 R_{3}^{2}+\left(R_{1}+H_{1}\right)^{2}\right]
$$

Радиус частицы конденсированного оксида является неизвестным и находится итерационным способом при помощи численного решения уравнений (1)-(4) при известном радиусе капли алюминия и содержании алюминия в агломерате, а также известной массе частиц окиси.

Конденсация или испарение частицы учитываются при помощи постановки граничных условий нормального отсоса (конденсация) или вдува (испарение) с постоянной скоростью 
на поверхности частицы. Распределение температуры газа находится из решения краевой задачи для уравнений движения и теплопереноса в области вне тела частицы. Считая, что процесс конденсации и испарения происходит в квазистационарном режиме, скорость отсоса или вдува вычисляется из условия теплового баланса на поверхности частицы, которое выражает собой равенство теплового потока теплоте фазового превращения. Часть поверхности частицы, занятая нашлепкой окиси, исключается из процесса горения (испарения). На поверхности частицы конденсированного оксида используются условия непротекания и прилипания.

\section{3. Метод локальной гидродинамики}

Рассматривается обтекание системы, состоящей из двух пересекающихся сфер, поступательным стоксовым потоком со скоростью $U$ и вязкостью $\mu$. При обтекании тела вращения любой формы при малых числах Рейнольдса, произвольно ориентированном в поступательном потоке, сила сопротивления представляется в виде

$$
\mathbf{F}=\left(F_{\|} \cos \varphi\right) \boldsymbol{\tau}+\left(F_{\perp} \sin \varphi\right) \mathbf{n},
$$

где $\boldsymbol{\tau}$ - единичный вектор, направленный вдоль оси тела; $\mathbf{n}$ - единичный вектор, лежащий в плоскости вращения тела; $\varphi$ - угол между осью тела вращения и направлением набегающего потока. Величина силы сопротивления $F=\mathbf{F} \cdot \mathbf{i}$, где $\mathbf{i}-$ единичный вектор, направленный вдоль потока, находится из соотношения

$$
F=F_{\|} \cos ^{2} \varphi+F_{\perp} \sin ^{2} \varphi
$$

Для вычисления силы сопротивления тела вращения любой формы, произвольно ориентированного в поступательном стоксовом потоке, достаточно знать величину этой силы только для двух частных пространственных положений тела. При этом осевая $F_{\|}$и нормальная $F_{\perp}$ составляющие силы определяются как теоретическим, так и экспериментальным способом. В частности, для гантелевидной частицы, состоящей из двух соприкасающихся сфер равного радиуса $a$, составляющие силы находятся из соотношения $F=12 \pi \mu a U \lambda$, где $\lambda_{\|}=0.645$ и $\lambda_{\perp}=0.716$ (сила сопротивления равняется сумме сопротивлений двух изолированных сфер радиусом $a$ ).

В рамках стоксового приближения сила сопротивления, действующая на составную частицу со стороны жидкости за счет вязкости, рассчитывается с помощью метода локальной гидродинамики. Сила сопротивления частицы сложной формы представляется в виде суммы сил сопротивления двух сферических поверхностей. Поверхность частицы разбивается на малые площадки, и вычисляется сила, действующая со стороны потока на каждую площадку. Сила сопротивления частицы находится как сумма найденных сил по всей поверхности.

Для нахождения сопротивления составной частицы используется решение задачи о стоксовом обтекании сферы потоком вязкой несжимаемой жидкости. Вычисляются силы сопротивления при стоксовом обтекании сферы радиусом $R_{1}$ и сферы радиусом $R_{2}$, и учитывается лишь те части поверхности составной частицы, которые находятся в контакте с жидкостью $\left(S=S_{1} \cup S_{2}\right)$. Сила сопротивления представляется в виде $F=F_{1}+F_{2}$, а силы $F_{1}$ и $F_{2}$ определяются как проекции гидродинамических сил, действующих на поверхности $S_{1}$ и $S_{2}$, на направление потока

$$
F_{1}=\int_{S_{1}}\left(\tau_{r r} \cos \varphi-\tau_{r \varphi} \sin \varphi\right) \mathrm{d} s, \quad F_{2}=\int_{S_{2}}\left(\tau_{r r} \cos \varphi-\tau_{r \varphi} \sin \varphi\right) \mathrm{d} s
$$

Нормальные и касательные компоненты тензора вязких напряжений на элементе поверхности вычисляются при помощи соотношений для напряжений на поверхности сферы при ее стоксовом обтекании (используются сферические координаты) [6] 


$$
\tau_{r r}=\frac{3}{2} \mu \frac{U}{a} \cos \varphi, \quad \tau_{r \varphi}=-\frac{3}{2} \mu \frac{U}{a} \sin \varphi
$$

\section{4. Метод решения}

Моделирование обтекания составной частицы в различных условиях проводится в рамках единого методологического подхода (невязкое/вязкое течения, течения при конечных числах Рейнольдса, учет вращения частицы). Численная модель строится на основе решения нестационарных уравнений Навье - Стокса для вязкого сжимаемого газа в трехмерной постановке на неструктурированных сетках. Схему решения задачи и связи между подзадачами поясняет рис. 3 .

Для дискретизации основных уравнений используется метод конечных объемов на неструктурированных сетках и среднемедианный контрольный объем [36]. Интегрирование по времени проводится методом Рунге-Кутты 3-го порядка. Дискретизация невязких потоков осуществляется при помощи схемы MUSCL (Monotonic Upstream Schemes for Conservation Laws, монотонная противопоточная схема для законов сохранения), а вязких потоков - центрированная схема 2-го порядка точности. Схема MUSCL позволяет повысить порядок аппроксимации по пространственным переменным без потери монотонности решения, удовлетворяет условию TVD (Total Variation Diminishing) и представляет собой комбинацию центрированных конечных разностей 2-го порядка и диссипативного члена, для переключения между которыми служит ограничитель потока, построенный на основе характеристических переменных. Нахождение градиента и псевдолапласиана в серединной точке грани контрольного объема производится на основе соотношений, приспособленных для расчетов на сильно растянутых сетках, используемых в пограничном слое [36]. Для решения системы разностных уравнений используется геометрический многосеточный метод [37]. Система сеток различной разрешающей способности строится при помощи метода схлопывающихся граней (Edge-Collapsing Method).

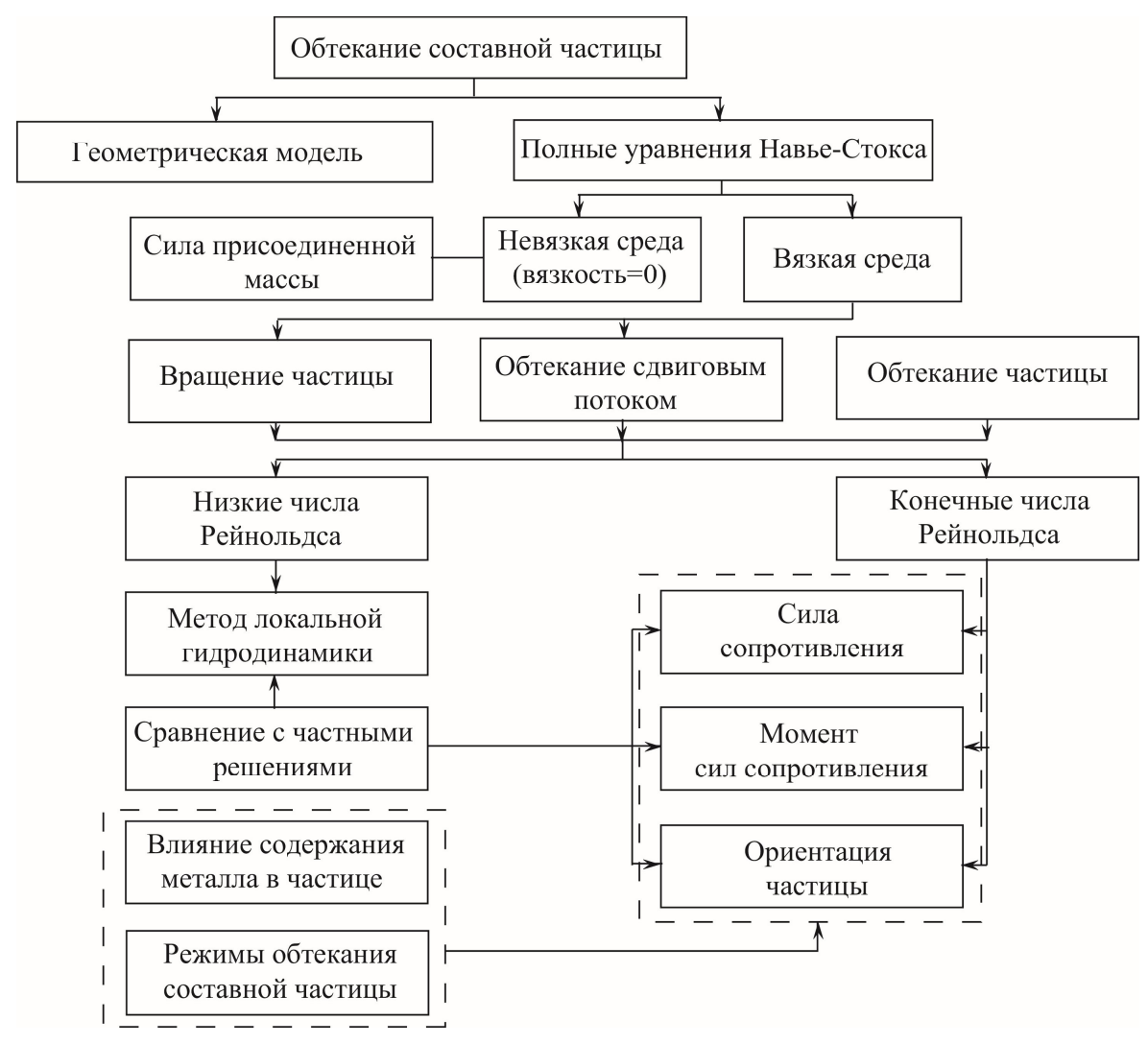

Рис. 3. Схема решения задачи и связи между подзадачами 
Для моделирования невязких течений коэффициенту вязкости присваивается нулевое значение. Для обеспечения устойчивого численного счета при моделировании низкоскоростных течений в рамках модели сжимаемого газа используется блочный метод предобусловливания Якоби [38].

Начало координат располагается в центре капли металла. Протяженность расчетной области в продольном направлении равняется $L_{x}=L_{x 1}+L_{x 2}$ ( $L_{x 1}$ перед частицей и $L_{x 2}$ позади частицы), а в направлении осей $y$ и $z-L_{y}$ и $L_{z}$. Расчеты проводятся при числах Рейнольдса, соответствующих различным режимам течения около частицы. Для каждого режима течения общее число узлов и разрешение сетки в пристеночной области подбирается исходя из значения числа Рейнольдса.

Характерный размер ячеек в пристеночной области составляет $g$, а коэффициент увеличения характерного размера ячейки в пограничном слое при переходе к следующему слою ячеек равняется $k$ (в пристеночной области для размещения узлов сетки применяется закон геометрической прогрессии). В частности, при $\mathrm{Re}=300$ полагается, что $g=0.005$ и $k=1.08$. При этом общее число узлов расчетной сетки составляет $9.6 \times 10^{5}$. Шаг интегрирования по времени полагается равным $\Delta t=0.04 R / v$. Делается приблизительно 8000 шагов по времени.

На входной границе расчетной области задается скорость невозмущенного потока. Скорость невозмущенного потока выбирается в соответствии с заданным числом Рейнольдса. На поперечных гранях используются условия скольжения. На поверхности частицы фиксируется температура, а для тангенциальной и нормальной компонент скорости используются условия прилипания и непротекания.

\section{5. Результаты расчетов}

Рассматривается обтекание двойной сферы потоком несжимаемой жидкости при различных числах Рейнольдса и углах сопряжения двух сфер, соответствующих капли металла и присоединенной частицы окиси.

\section{1. Обтекание невязкой жидкостью}

Линии тока при обтекании двойной сферы потоком невязкой несжимаемой жидкости показывает рис. 4. Приведенные картины линий тока соответствуют фиксированному углу пересечения и различным радиусам сфер.

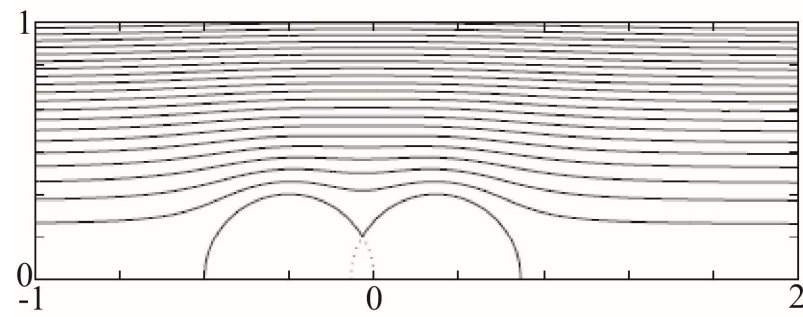

a)

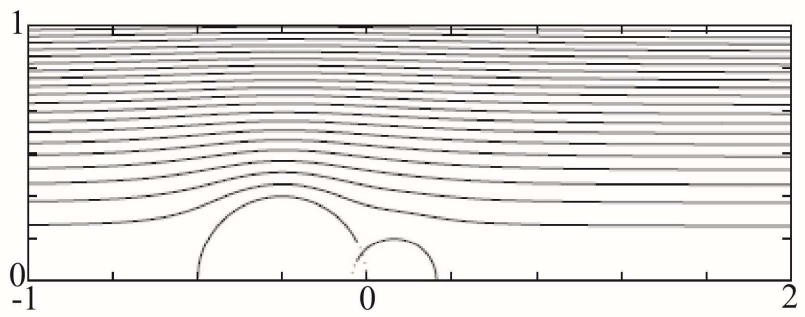

б)

Рис. 4. Линии тока при обтекании двойной сферы невязкой жидкостью для угла пересечения сфер $\theta=60^{\circ}$ при $R_{2} / R_{1}=1($ a) и 2 (б)

Зависимость присоединенной массы двойной сферы от отношения радиусов двух сфер показывает рис. 5 для различных углов пересечения сфер. Результаты расчетов нормируются на объем двойной сферы $\left(V=V_{1}+V_{2}\right)$. Присоединенная масса сферы равняется половине ее массы. Присоединенная масса двойной сферы при фиксированном угле пересечения сфер монотонно уменьшается, достигая минимума при $R_{2} / R_{1}=1$, а затем монотонно возрастает. 




Рис. 5. Присоединенная масса двойной сферы при $\theta=90^{\circ}(1) ; 60^{\circ}$ (2); $45^{\circ}$ (3)

\section{2. Обтекание при низких числах Рейнольдса}

Линии тока при обтекании двойной сферы вязкой несжимаемой жидкостью в различных направлениях показывают рис. 6 и рис. 7. Приведенные картины линий тока соответствуют фиксированному углу пересечения и различным радиусам сфер.

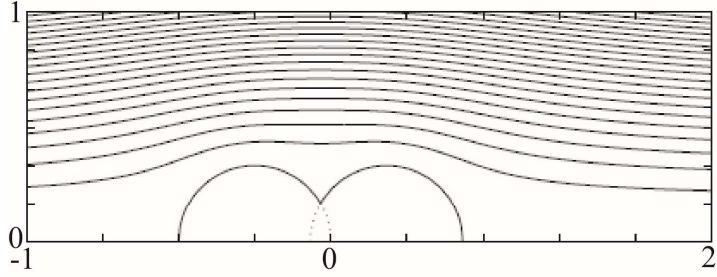

a)

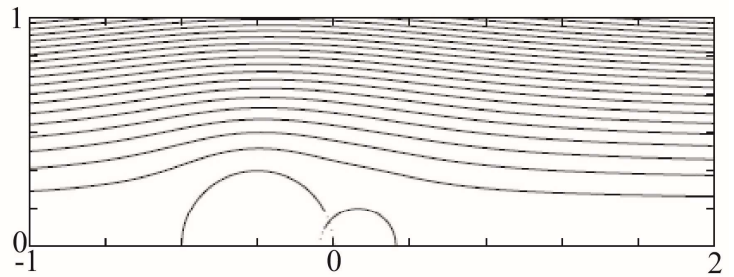

б)

Рис. 6. Линии тока при обтекании двойной сферы вязкой несжимаемой жидкостью в направлении, параллельном линии, соединяющей центры двух сфер, для угла пересечения сфер $\theta=60^{\circ}$ при $R_{2} / R_{1}=1(a)$ и 2 (б)

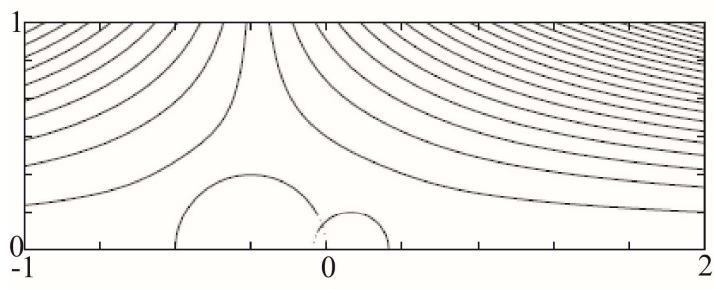

a)

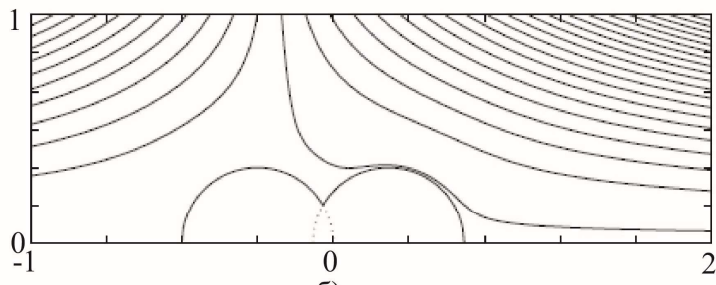

б)

Рис. 7. Линии тока при обтекании двойной сферы вязкой несжимаемой жидкостью в направлении, нормальном линии, соединяющей центры двух сфер, для угла пересечения сфер $\theta=60^{\circ}$ при $R_{2} / R_{1}=1$ (a) и 2 (б)

Зависимости силы сопротивления двойной сферы при ее стоксовом обтекании в направлении, параллельном и нормальном линии, соединяющей центры сфер, от отношения радиусов двух сфер показывает рис. 8 для различных углов пересечения сфер. Результаты расчетов нормируются на силу сопротивления, соответствующую стоксовому режиму течения со скоростью $U$ около сферы радиусом $R=R_{1}+R_{2}$ и площадью миделевого сечения $S=\pi\left(R_{1}+R_{2}\right)^{2}$. В стоксовом режиме течения сила сопротивления сферы радиусом $R$ в жидкости с вязкостью $\mu$ находится из соотношения $F_{S}=6 \pi \mu U R$. 
Сила сопротивления двойной сферы при ее обтекании в направлении, параллельном линии, соединяющей центры двух сфер, при фиксированном угле пересечения сфер монотонно уменьшается, достигая минимума при $R_{2} / R_{1}=1$, а затем монотонно возрастает. Минимальное значение силы сопротивления наблюдается для сфер одинакового радиуса $\left(R_{2} / R_{1}=1\right)$ при $\theta=90^{\circ}$. Случай $R_{2}=0$ соответствует стоксовому обтеканию сферы. При $R_{2} / R_{1} \gg 1$ нормированная сила сопротивления стремится к постоянному значению. Для $\theta=180^{\circ}$ расчеты достаточно хорошо согласуются с данными, приведенными в работе [17] для двух соприкасающихся сфер. При обтекании двойной сферы в направлении, нормальном линии, соединяющей центры двух сфер, сила сопротивления монотонно возрастает для всех значений $R_{2} / R_{1}$ и углов пересечения сфер.

Зависимости момента сил сопротивления вращению двойной сферы от отношения радиусов двух сфер показывает рис. 9 для различных углов пересечения сфер. Результаты расчетов нормируются на момент сил сопротивления, соответствующий медленному вращению сферы радиусом $R=R_{1}+R_{2}$ и объемом $V=V_{1}+V_{2}$ с угловой скоростью $\omega$. В стоксовом режиме течения момент сил сопротивления вращению сферы радиусом $R$ в жидкости с вязкостью $\mu$ находится из соотношения $L_{S}=8 \pi \mu \omega R^{3}$.

Момент сопротивления двойной сферы при фиксированном угле пересечения сфер монотонно уменьшается, достигая минимума при $R_{2} / R_{1}=1$, а затем монотонно возрастает. Минимальное значение момента сопротивления наблюдается для сфер одинакового радиуса $\left(R_{2} / R_{1}=1\right)$ при $\theta=90^{\circ}$. Случай $R_{2}=0$ соответствует стоксовому обтеканию сферы. При $R_{2} / R_{1} \gg 1$ нормированный момент сопротивления стремится к постоянному значению. Для $\theta=180^{\circ}$ расчеты достаточно хорошо согласуются с данными, приведенными в работе [17] для двух соприкасающихся сфер. Нижняя и верхняя границы момента сопротивления удовлетворяют соотношению

$$
V_{1} \leq \frac{L}{8 \pi \mu \omega} \leq V_{1}+V_{2}
$$


Рис. 8. Силы сопротивления двойной сферы при ее обтекании в направлении, параллельном (a) и нормальном (б) линии, соединяющей центры двух сфер, при $\theta=90^{\circ}(1) ; 60^{\circ}(2)$; $45^{\circ}(3)$

\section{3. Вращение в сдвиговом потоке}

Рассматривается обтекание и вращение двух соприкасающихся сфер в потоке со сдвигом скорости. Сдвиг скорости формируется за счет движения нижней и верхней границ расчетной области параллельно друг другу с постоянной скоростью $U$ в противоположных направлениях. Расстояние между нижней и верхней границами расчетной области составляет $Z$. Сдвиг скорости определяется соотношением $s=2 U / Z$. Картина течения около системы сфер, а также сила и момент сил сопротивления зависят от ориентации частиц по отношению 
к скорости набегающего потока. Угол между линией, соединяющей центры двух сфер, и направлением набегающего потока обозначается через $\varphi$. Число Рейнольдса находится из соотношения $\mathrm{Re}=s a^{2} / v$. Отношение плотностей материала частицы и жидкости полагается равным 4.

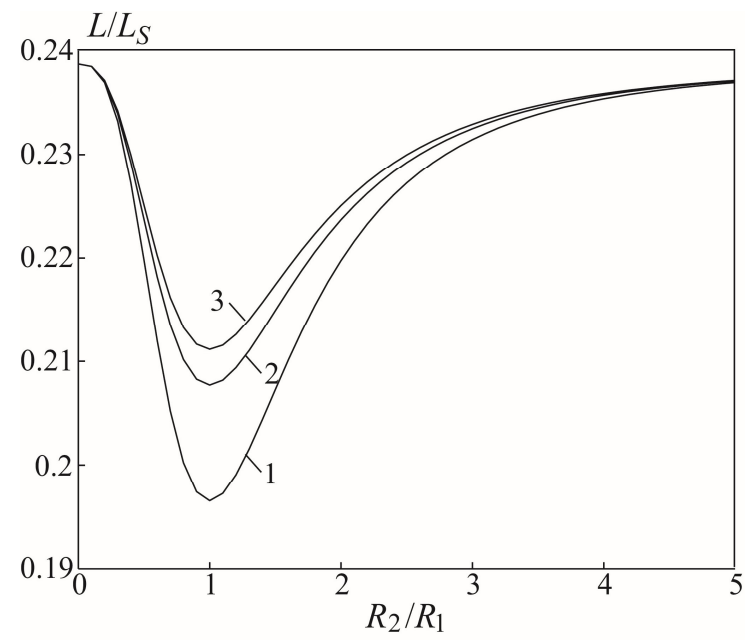

Рис. 9. Момент сил сопротивления двойной сферы при $\theta=90^{\circ}(1)$; $60^{\circ}(2) ; 45^{\circ}(3)$

В момент времени $t=0$ пластины начинают движение в противоположных направлениях с равной по величине скоростью, а частица ориентирована по направлению набегающего потока $(\varphi=0)$. На поверхности сферы применяются условия непротекания и прилипания для нормальной и тангенциальной компонент скорости. В продольном направлении используются периодические граничные условия.

Результаты расчетов сравниваются с точным решением задачи, приведенным в работе [16] (при $\operatorname{Re} \ll 1$ ). В системе координат, связанной с частицей, сила и момент вращения находятся из соотношений

$$
F=\pi \mu \frac{a^{2}}{2} s\left(h_{1}+h_{2}\right) \sin (2 \varphi), \quad L=\frac{1}{2} s[1+C \cos (2 \varphi)]
$$

Постоянные $h_{1}$ и $h_{2}$ зависят от отношения радиусов двух сфер $\left(h_{1}=4.463\right.$ и $h_{2}=7.767$ для сфер одинакового размера). Постоянная $C$ находится из соотношения

$$
C=\frac{r_{e}^{2}-1}{r_{e}^{2}+1},
$$

в котором под $r_{e}$ понимается эквивалентный радиус системы $\left(r_{e}=1.982\right.$ для сфер одинакового размера). При этом $F$ представляет собой силу, нормальную линии, соединяющей центры двух сфер, а $L$ - момент относительно оси, нормальной плоскости вращения. Система испытывает максимальную подъемную силу и минимальный вращающий момент, когда линия, соединяющая центры двух сфер, оказывается параллельной направлению набегающего потока $\left(\varphi=0^{\circ}\right)$, и минимальную силу и максимальный момент - в случае, когда набегающий поток оказывается нормальным к этой линии $\left(\varphi=90^{\circ}\right)$.

Результаты расчетов показывает рис. 10 для полностью развитого течения между пластинами при $R e=0.06$. Зависимость силы, действующей на частицу, от ориентации частицы носит периодический характер с постоянной амплитудой (после небольшой задержки, связанной с установлением поле течения между пластинами). Наблюдается достаточно хорошее согласование расчетных результатов с точным решением задачи, приведенным в работе [16]. Фазовый сдвиг между точным и численным решениями составляет около $0.014 \pi$, а рассогласование по амплитуде - около $1 \%$. 



Рис. 10. Сила ( $a$ ) и момент (б), действующие на двойную сферу. Сплошные линии соответствуют результатам расчетов, а пунктирные линии - точному решению задачи [16]

Число Рейнольдса оказывает существенное влияние на результаты расчетов, поскольку подъемная сила является пропорциональной $\mathrm{Re}^{1 / 2}$. Величина подъемной силы Саффмана для частицы сферической формы находится из соотношения $F_{S}=1.29 \mu a^{2} s \operatorname{Re}^{1 / 2}$ (при $\varphi=0^{\circ}$ ). По результатам численных расчетов $F=0.5 \mu a^{2} s$ при $\mathrm{Re}=0.03$ и $F=0.9 \mu a^{2} s$ при $\operatorname{Re}=0.06$, в то время как теоретические оценки силы Саффмана для сферы дают $F_{S}=0.22 \mu a^{2} s$ при $\operatorname{Re}=0.03$ и $F_{S}=0.32 \mu a^{2} s$ при $\mathrm{Re}=0.03$. Увеличение числа Рейнольдса приводит к увеличению фазового сдвига между точным и численным решением.

\section{4. Вращательное движение присоединенной частицы}

Для учета вращательного движения присоединенной частицы по поверхности капли металла вводится локальная система координат, и находятся проекции силы, действующей на присоединенную частицу (рис. 11). Составляющие силы, определяющей вращение присоединенной частицы вокруг капли металла, находятся из соотношения

$$
F_{\tau}=F_{x} \cos \theta-F_{y} \sin \theta,
$$

где $\theta$ - угол, определяющий ориентацию составной частицы относительно потока.

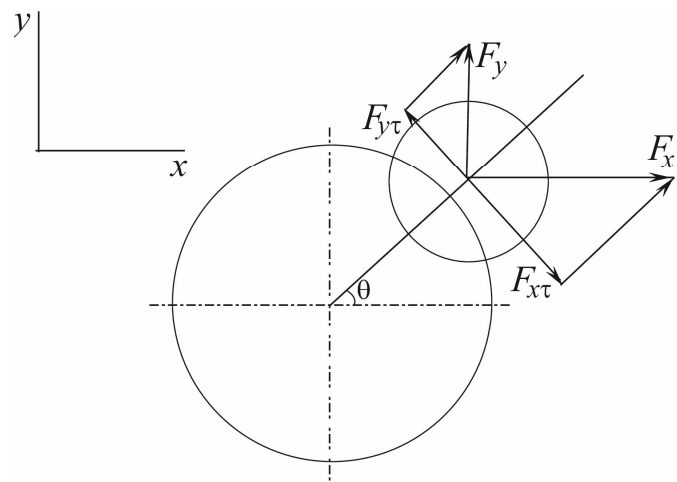

Рис. 11. Силы, приложенные к присоединенной частице

Уравнение вращения имеет вид

$$
\frac{\mathrm{d}^{2} \theta}{\mathrm{d} t^{2}}\left(m_{2}+\rho_{g} V_{2} K\right) l^{2}=F_{\tau} l-k d^{2} \frac{\mathrm{d} \theta}{\mathrm{d} t},
$$

где $m_{2}$ и $V_{2}$ - масса и объем присоединенной частицы; $l$ - расстояние между центрами масс двух сфер, составляющих частицу. Слагаемое $\rho_{g} V_{2} K$ позволяет учесть эффект присоединенной массы. Член $k d^{2}(\mathrm{~d} \theta / \mathrm{d} t)$ учитывает силу трения, возникающую при скольжении присоединенной частицы по поверхности капли металла. 
Коэффициент трения оценивается, исходя из рассмотрения движения тела по поверхности жидкой сферы (рис. 12). В движение вовлекаются лишь приграничные слои жидкости, внутренние слои остаются неподвижными. Оценка коэффициента трения дает

$$
\tau=\mu \frac{\partial u}{\partial r} \sim \mu \frac{U}{w R} \sim \mu \frac{\theta}{w} S \sim \mu \frac{\mathrm{d}^{2}}{w} \frac{\mathrm{d} \theta}{\mathrm{d} t},
$$

где $\mu$ - динамическая вязкость газа; $w R$ - толщина вовлеченных в движение слоев жидкости; $S$ - площадь соприкосновения двух сфер; $d$ - диаметр окружности, по которой пересекаются основная и присоединенная частицы.

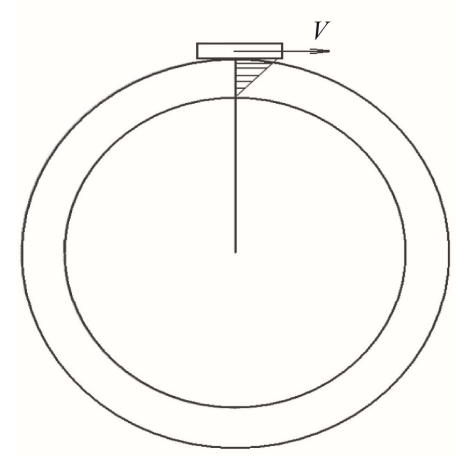

Рис. 12. Профиль скорости при движении тела по поверхности сферы

Для расчетов применяется сферическая система координат. Уравнения, описывающие вращательное движение присоединенной частицы оксида по поверхности капли металла, имеют вид

$$
\frac{\mathrm{d} \theta}{\mathrm{d} t}=\omega, \quad m_{2} l \frac{\mathrm{d} \omega}{\mathrm{d} t}=F_{\tau}
$$

где $m_{2}$ - масса присоединенной частицы; $\omega$ - угловая скорость частицы. В качестве начальных условий в момент времени $t=0$ задается угол $\theta_{0}$, характеризующий начальную ориентацию присоединенной частицы, и нулевая угловая скорость вращения.

Изменение угла ориентации присоединенной частицы во времени показывает рис. 13.

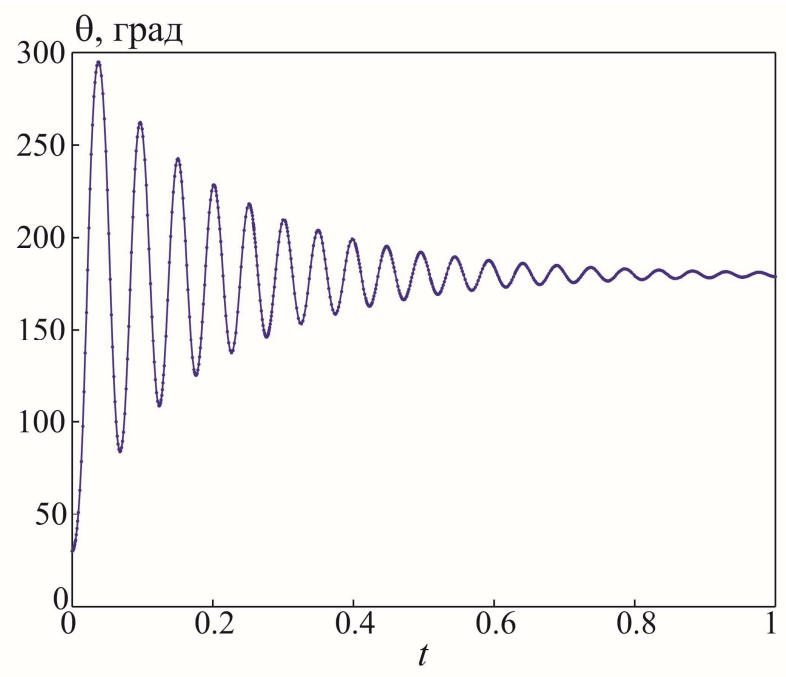

Рис. 13. Изменение угла, характеризующего положение присоединенной частицы на поверхности капли металла, во времени 
В случае свободного скольжения $(k=0)$ присоединенная частица совершает бесконечное колебательное движение, а в случае с трением $(k \neq 0)$ присоединенная частица через некоторое время релаксации занимает положение устойчивого равновесия и перестает вращаться. Фазовый портрет системы показывает рис. 14.

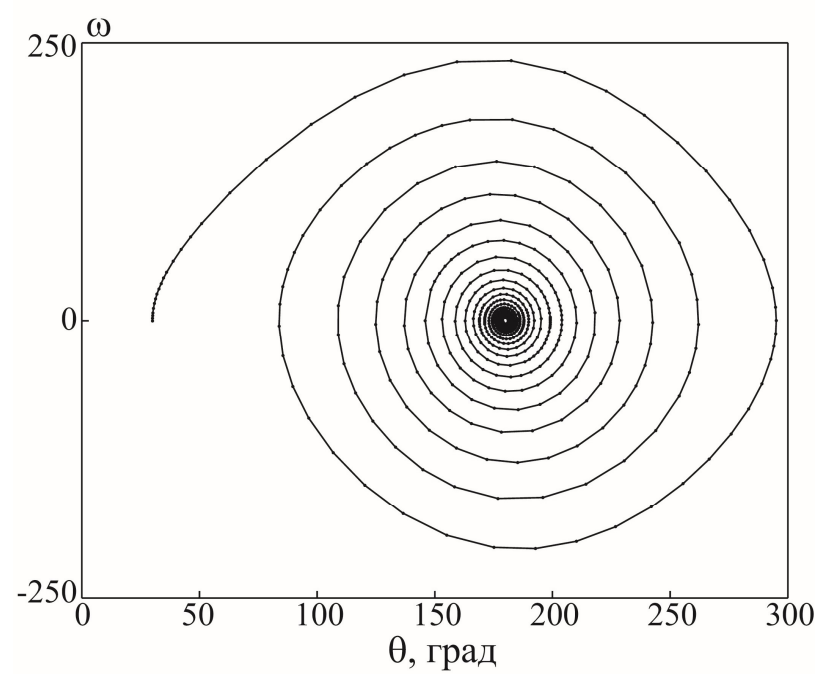

Рис. 14. Фазовый портрет скольжения присоединенной частицы по поверхности основной частицы

\section{5. Обтекание при умеренных числах Рейнольдса}

Линии тока при обтекании составной частицы потоком вязкой несжимаемой жидкости показаны на рис. 15 при различных числах Рейнольдса. В целом, картина обтекания составной частицы соответствует теоретическим представлениям об изменениях картины течения около тела вращения при увеличении числа Рейнольдса.



Рис. 15. Линии тока около составной частицы при $\mathrm{Re}=5$ (a); 20 (б); 40 (в); 60 (2); 80 (d); $100(e)$

Характерной чертой в распределениях трения и вихря скорости по поверхности составной частицы является наличие двух максимумов этих характеристик (рис. 16). Один их них соответствует поверхности частицы алюминия, другой - поверхности нашлепки окиси. Влияние вдува с поверхности частицы незначительно сказывается на распределении давления по ее поверхности (рис. 17). Тепловой поток уменьшается, начиная от передней критической точки, достигает минимума при значении полярного угла, соответствующего сопряжению капли алюминия и частицы окиси, что примерно соответствует точке отрыва пограничного слоя, и далее вниз по потоку резко возрастает. Увеличение скорости вдува с 0.1 до 0.4 
приводит почти к четырехкратному уменьшению теплового потока к поверхности капли алюминия (рис. 18), в то время как тепловой поток к поверхности частицы конденсированного оксида, напротив, увеличивается (примерно в 1.5 раза). Полученные распределения локальных характеристик течения качественно согласуются с результатами, приведенными в работе [26] (количественное сравнение результатов затруднительно в связи с недостатком данных о входных параметрах задачи).
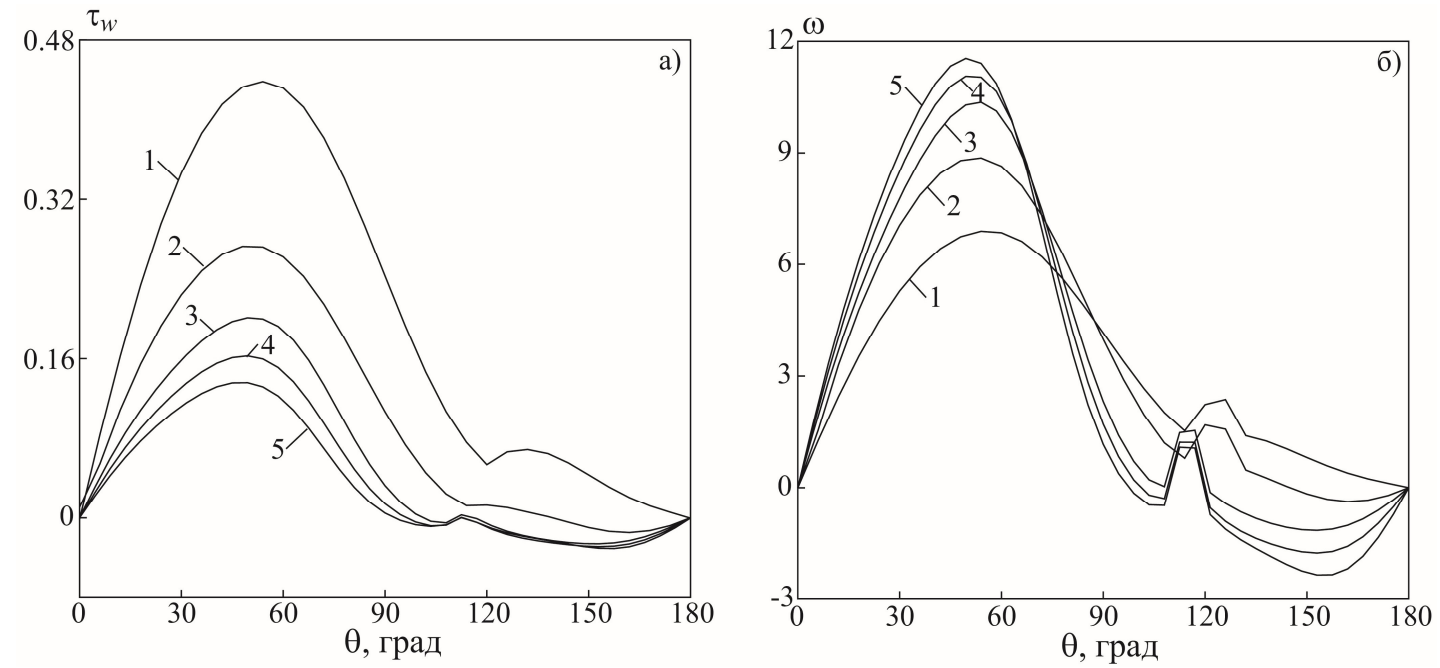

Рис. 16. Распределения напряжения трения ( $a$ ) и вихря скорости (б) по поверхности составной частицы при $\mathrm{Re}=20$ (1); 40 (2); 60 (3); 80 (4); 100 (5)



Рис. 17. Распределения давления по поверхности составной частицы (число Рейнольдса равно 80) при $v_{w}=0.1$ (1); 0.4 (2)

При $C_{1}>0.4$ составная частица ориентируется нашлепкой к потоку, а при $C_{1}<0.4$ разворачивается на $180^{\circ}$ (открытая поверхность капли алюминия к потоку) [26]. Продукты испарения встречаются с набегающим потоком в узкой зоне вблизи поверхности частицы. При ориентации частицы нашлепкой к потоку продукты испарения создают завесу, препятствующую проникновению потока к открытой поверхности алюминия в кормовой части. В кормовой области имеется низкоскоростное циркуляционное течение. Такое распределение скорости оказывает влияние на горение составных агломератов. В этом случае гетерогенное окисление протекает слабо, а процесс горения частицы определяется кипением смеси $\mathrm{Al}+\mathrm{Al}_{2} \mathrm{O}_{3}$ на границе контакта. При повороте агломерата открытой поверхностью алюминия к потоку картина обтекания изменяется. Поток окислителя к поверхности алюминия увеличивается, а к поверхности нашлепки уменьшается. 


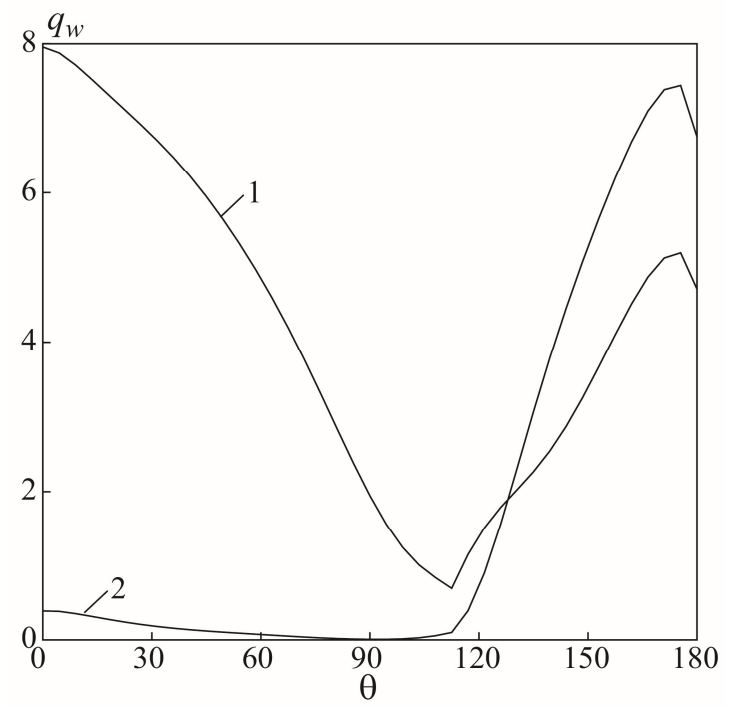

Рис. 18. Распределения теплового потока по поверхности составной частицы (число Рейнольдса равно 80) при $v_{w}=0.1$ (1); 0.4 (2)

При обтекании составной частицы двухфазным потоком, содержащим частицы высокодисперсного оксида, имеет место осаждение частиц из двухфазного потока на поверхность нашлепки. Давление на поверхности агломерата распределено неравномерно. При определенных условиях частица алюминия вдавливается в частицу оксида, что приводит к захлопыванию нашлепки. В этом случае возможно положение агломерата с частицей алюминия внутри. Частицы такого вида довольно часто встречаются в конденсированных продуктах сгорания металлизированных топлив.

Зависимость коэффициента сопротивления от содержания алюминия в составной частице носит немонотонный характер (рис. 19).



Рис. 19. Зависимость коэффициента сопротивления составной частицы от содержания алюминия

В области малых чисел Рейнольдса сопротивление частицы определяется, в основном, вязкими силами. После отрыва потока сопротивление частицы обусловлено не трением в пограничном слое, а разностью статических давлений в лобовой и кормовой частях частицы. Коэффициент сопротивления при малых скоростях обтекания $(\operatorname{Re}<180)$ со временем изменяется монотонным образом, принимая при $t>30$ постоянное значение, зависящее от числа 
Рейнольдса. При $\operatorname{Re}>250$ наблюдаются периодические изменения продольной составляющей скорости в следе за частицей, которые, в свою очередь, приводят к колебаниям коэффициентов сопротивления и подъемной силы. Смена режимов течения жидкости около частицы с каплей конденсированного оксида происходит при больших числах Рейнольдса, чем при обтекании твердой сферы. Так, переход от стационарного симметричного обтекания частицы к стационарному несимметричному режиму течения происходит при $\operatorname{Re} \sim 250$ (для сферы $\mathrm{Re} \sim 210$ ), а переход от стационарного несимметричного режима течения к нестационарному несимметричному обтеканию начинается примерно при $\operatorname{Re} \sim 410$ (для сферы $\operatorname{Re} \sim 380$ ).

\section{6. Заключение}

Построена геометрическая модель капли металла с присоединенной к ней частицей конденсированного оксида. В модели радиус частицы конденсированного оксида является неизвестным и находится итерационным способом при известном радиусе капли алюминия и содержании алюминия в агломерате, а также известной массе частиц окиси. Подключение модели изменения массы частиц позволит использовать разработанную модель для исследования горения капель в газодинамических трактах РДТТ.

На основе общего методологического подхода проведено численное моделирования течения около составной частицы. Полученные результаты позволяют оценить влияние геометрии частицы и параметров набегающего потока на картину течения около составной частицы (поле течения, размер отрывной зоны, координаты точки отрыва потока), а также локальные (распределения давления, напряжения поверхностного трения, теплового потока) и интегральные (коэффициенты сопротивления и теплоотдачи) характеристики течения. Зависимости присоединенной массы и момента сил сопротивления двойной сферы от угла сопряжения сфер носят немонотонный характер, достигая минимума при равных размерах капли металла и частицы присоединённой окиси. Немонотонный характер имеет также зависимость коэффициента сопротивления составной частицы от содержания алюминия. Характерной чертой в распределениях трения и вихря скорости по поверхности составной частицы является наличие двух максимумов этих характеристик, один из которых соответствует капле металла, а другой - частице окиси. Смена режимов течения жидкости около частицы с каплей конденсированного оксида происходит при больших числах Рейнольдса, чем при обтекании твердой сферы.

Результаты численных расчетов представляются полезными при корректировке зависимостей для коэффициентов сопротивления и теплоотдачи частиц-агломератов несферической формы при относительных скоростях обтекания, соответствующих образованию отрывных зон.

\section{Литература}

1. Emelyanov V.N., Teterina I.V., Volkov K.N., Garkushev A.U. Pressure oscillations and instability of working processes in the combustion chambers of solid rocket motors // Acta Astronautica. 2017. Vol. 135. Pp. 161-171.

2. Price E.W. Combustion of metallized propellants // Progress in Astronautics and Aeronautics: Fundamentals of Solid Propellant Combustion. 1985. Vol. 90. Pp. 479-513.

3. Бабук В.А., Белов В.П., Ходосов В.В., Шелухин Г.Г. Исследование структуры агломератов при горении алюминизированных смесевых конденсированных систем // Физика горения и взрыва. 1988. T. 24. № 5. C. 52-56.

4. Eisel J.L., Brown B.G., Price E.W. Pressure, velocity and geometry effect on $\mathrm{Al}_{2} \mathrm{O}_{3}$ produced during aluminized propellant combustion // AIAA Journal. 1975. Vol. 13. No. 7. Pp. 913-917.

5. Olsen S.E., Beckstead M.W. Burn time measurements of single aluminum particles in steam and carbon dioxide mixtures // Journal of Propulsion and Power. 1996. Vol. 12. No. 4. Pp. 662-671.

6. Волков К.Н., Емельянов В.Н. Течения газа с частицами. М.: Физматлит, 2008. 598 с. 
7. Carrasco B., Garcia de la Torre J., Zipper P. Calculation of hydrodynamic properties of macromolecular bead models with overlapping spheres//European Biophysics Journal. 1999. Vol. 28. Pp. 510-515.

8. Strobl S., Formella A., Poschel T. Exact calculation of the overlap volume of spheres and mesh elements // Journal of Computational Physics. 2016. Vol. 311. Pp. 158-172.

9. Leith B. Drag on nonspherical objects//Aerosol Science and Technology. 1987. Vol. 6. No. 2. Pp. 153161.

10. Wang-yi W., Xiao-yi H. The stokes flow of the rotating double spheres and multiple spheres // Applied Mathematics and Mechanics. 1987. Vol. 8. Pp. 123-136.

11. Folkersma R., Stein H.N., van de Vosse F.N. Hydrodynamic interactions between two identical spheres held fixed side by side against a uniform stream directed perpendicular to the line connecting the spheres' centres // International Journal of Multiphase Flow. 2000. Vol. 26. No. 5. Pp. 877-887.

12. Ebrahimi M., Crapper M., Ooi J. Numerical and experimental study of horizontal pneumatic transportation of spherical and low-aspect-ratio cylindrical particles // Powder Technology. 2016. Vol. 293. Pp. 48-59.

13. Liang S.-C., Hong T., Fan L.-S. Effects of particle arrangements on the drag force of a particle in the intermediate flow regime // International Journal of Multiphase Flow. 1996. Vol. 22. No. 2. Pp. 285 306.

14. Verma P.L., Govardhan M. Flow behind bluff bodies in side-by-side arrangement // Journal of Engineering Science and Technology. 2011. Vol. 6. No. 6. Pp. 745-768.

15. Cichocki B., Hinsen K. Stokes drag on conglomerates of spheres // Physics of Fluids. 1995. Vol. 7. No. 2. Pp. 285-291.

16. Nir A., Acrivos A. On the creeping motion of two arbitrary-sized touching spheres in a linear shear field // Journal of Fluid Mechanics. 1973. Vol. 59. P. 209-223.

17. Derksen J.J. Flow-induced forces in sphere doublets // Journal of Fluid Mechanics. 2008. Vol. 608. Pp. 337-356.

18. Derksen J.J., Larsen R.A. Drag and lift forces on random assemblies of wall-attached spheres in lowReynolds-number shear flow // Journal of Fluid Mechanics. 2011. Vol. 673. Pp. 1-27.

19. Zhao L. Hydrodynamics of highly viscous flow past a compound particle: analytical solution // Fluids. 2016. Vol. 1. 36 (15 pages).

20. Daripa P., Pallaniaappan D. Generalized circle and sphere theorems for inviscid and viscous flows // SIAM Journal of Applied Mathematics. 2001. Vol. 63. No. 2. Pp. 514-540.

21. Schneider J.C., O’Neill M.E., Brenner H. On the slow viscous rotation of a body straddling the interface between two immiscible semi-infinite fluids // Mathematika. 1973. Vol. 20. No. 2. Pp. 175-196.

22. Radchik A.V., Paley A.V., Smith G.B., Vagov A.V. Polarization and resonant absorption of intersecting cylinders and spheres // Journal of Applied Physics. 1994. Vol. 76. No. 8. Pp. 4827-4835.

23. Mansfield M.L., Douglas J.F., Garboczi E.J. Intrinsic viscosity and the electrical polarizability of arbitrarily shaped objects // Physics Review E. 2001. Vol. 64. 061401 (10 pages).

24. Бабук В.А., Будный Н.Л. Моделирование формирования оксида при горении металла агломератов // Химическая физика и мезоскопия. 2015. Т. 17. № 1. С. 39-50.

25. Бабук В.А., Низяев А.А. К вопросу о формировании «полых» агломератов при горении смесевых твердых топлив // Химическая физика и мезоскопия. 2016. Т. 18. № 1. С. 5-16.

26. Тененев В.А., Русяк И.Г., Горохов М.М. Численное исследование горе ния частиц алюминия в двухфазном потоке // Математическое моделирование. 1997. Т. 9. № 5. С. 87-96.

27. Гремячкин В.М. Теоретические модели горения металлических частиц // Физика аэродисперсных систем. 1987. Вып. 31. С. 41-52.

28. Стронгин М.П., Кошелев К.Б. Математическое моделирование нестационарных эффектов при обтекании одиночной горящей частицы // Физика горения и взрыва. 1993. Т. 29. № 3. С. 120 123. 
29. French A.D., Panelli M., Lorenzo G.D., Schettino A. Combustion instability and pressure oscillation numerical simulation in a solid rocket motor // AIAA Paper. 2017. No. 4952 (25 pages).

30. Volkov K.N. Combustion of single aluminium droplet in two-phase flow // Heterogeneous Combustion. Nova Science, 2010. Pp. 191-260.

31. King M.K. Aluminum combustion in solid rocket motor environment// Proceedings of the Combustion Institute. 2009. Vol. 32. No. 2. Pp. 2107-2114.

32. Yang J., Yoon W. Modeling of aluminum particle combustion with emphasis on the oxide effects and variable transport properties // Journal of Mechanical Science and Technology. 2010. Vol. 24. No. 4. Pp. 909-921.

33. Glorian J., Gallier S., Catoire L. On the role of heterogeneous reactions in aluminum combustion // Combustion and Flame. 2016. Vol. 168. Pp. 378-392.

34. Muller M., Davidenko D., Giovangigli V. Computational study of aluminum droplet combustion in different atmospheres // Proceedings of the 7th European Conference for Aeronautics and Space Sciences (EUCASS), 3-6 July 2017, Milan, Italy. 17 p.

35. Волков К.Н., Емельянов В.Н., Тетерина И.В., Яковчук М.С. Газовые течения в соплах энергоустановок. М.: Физматлит, 2016. 336 с.

36. Волков К.Н. Дискретизация уравнений Навье-Стокса на неструктурированной сетке при помощи метода контрольного объема и разностных схем повышенной разрешающей способности // Журнал вычислительной математики и математической физики. 2008. Т. 48. № 7. С. 1250 1273.

37. Волков К.Н. Многосеточные технологии для решения задач газовой динамики на неструктурированных сетках // Журнал вычислительной математики и математической физики. 2010. Т. 50. № 11. C. 1938-1952.

38. Волков К.Н. Блочное предобусловливание уравнений Эйлера и Навье-Стокса при моделировании низкоскоростных течений на неструктурированных сетках // Журнал вычислительной математики и математической физики. 2009. Т. 49. № 10. С. 1868-1884.

\section{References}

1. Emelyanov, V. N., Teterina, I. V., Volkov, K. N., Garkushev, A. U., "Pressure oscillations and instability of working processes in the combustion chambers of solid rocket motors," Acta Astronautica, Vol. 135, 2017, pp. 161-171.

2. Price, E. W., "Combustion of metallized propellants," Progress in Astronautics and Aeronautics: Fundamentals of Solid Propellant Combustion, Vol. 90, 1985, pp. 479-513.

3. Babuk, V. A., Belov, V. P., Hodosov, V. V., Sheluhin, G. G., "Issledovanie struktury aglomeratov pri gorenii aljuminizirovannyh smesevyh kondensirovannyh system," Fizika gorenija i vzryva, Vol. 24, No. 5, 1988, pp. 52-56.

4. Eisel, J. L., Brown, B. G., Price, E. W., "Pressure, velocity and geometry effect on $\mathrm{Al}_{2} \mathrm{O}_{3}$ produced during aluminized propellant combustion," AIAA Journal, Vol. 13, No. 7, 1975, pp. 913-917.

5. Olsen, S. E., Beckstead, M. W., "Burn time measurements of single aluminum particles in steam and carbon dioxide mixtures," Journal of Propulsion and Power, Vol. 12, No. 4, 1996, pp. 662-671.

6. Volkov, K. N., Emel'janov, V. N. Techenija gaza s chasticami (Gas flow with particles), M.: Fizmatlit, 2008. $598 \mathrm{~s}$.

7. Carrasco, B., Garcia de la Torre, J., Zipper, P., "Calculation of hydrodynamic properties of macromolecular bead models with overlapping spheres," European Biophysics Journal, Vol. 28, 1999, pp. 510 515 .

8. Strobl, S., Formella, A., Poschel, T., "Exact calculation of the overlap volume of spheres and mesh elements," Journal of Computational Physics, Vol. 311, 2016, pp. 158-172. 
9. Leith, B., "Drag on nonspherical objects," Aerosol Science and Technology, Vol. 6, No. 2, 1987, pp. 153-161.

10. Wang-yi, W., Xiao-yi, H., "The stokes flow of the rotating double spheres and multiple spheres," Applied Mathematics and Mechanics, Vol. 8, 1987, pp. 123-136.

11. Folkersma, R., Stein, H. N., van de Vosse, F. N., "Hydrodynamic interactions between two identical spheres held fixed side by side against a uniform stream directed perpendicular to the line connecting the spheres' centres," International Journal of Multiphase Flow, Vol. 26, No. 5, 2000, pp. 877-887.

12. Ebrahimi, M., Crapper, M., Ooi, J., "Numerical and experimental study of horizontal pneumatic transportation of spherical and low-aspect-ratio cylindrical particles," Powder Technology, Vol. 293, 2016, pp. 48-59.

13. Liang, S.-C., Hong, T., Fan, L.-S., "Effects of particle arrangements on the drag force of a particle in the intermediate flow regime," International Journal of Multiphase Flow, Vol. 22, No. 2, 1996, pp. 285-306.

14. Verma, P. L., Govardhan, M., "Flow behind bluff bodies in side-by-side arrangement," Journal of Engineering Science and Technology, Vol. 6, No. 6, 2011, pp. 745-768.

15. Cichocki, B., Hinsen, K., "Stokes drag on conglomerates of spheres," Physics of Fluids, Vol. 7, No. 2, 1995, pp. 285-291.

16. Nir, A., Acrivos, A., "On the creeping motion of two arbitrary-sized touching spheres in a linear shear field," Journal of Fluid Mechanics, Vol. 59, 1973, pp. 209-223.

17. Derksen, J. J., "Flow-induced forces in sphere doublets," Journal of Fluid Mechanics, Vol. 608, 2008, pp. 337-356.

18. Derksen, J. J., Larsen, R. A., "Drag and lift forces on random assemblies of wall-attached spheres in low-Reynolds-number shear flow," Journal of Fluid Mechanics, Vol. 673, 2011, pp. 1-27.

19. Zhao, L., "Hydrodynamics of highly viscous flow past a compound particle: analytical solution," Fluids, Vol. 1, No. 36, 2016. (15 pages).

20. Daripa, P., Pallaniaappan, D., "Generalized circle and sphere theorems for inviscid and viscous flows," SIAM Journal of Applied Mathematics, Vol. 63, No. 2, 2001, pp. 514-540.

21. Schneider, J. C., O’Neill, M. E., Brenner, H., "On the slow viscous rotation of a body straddling the interface between two immiscible semi-infinite fluids," Mathematika, Vol. 20, No. 2, 1973, pp. 175196.

22. Radchik, A. V., Paley, A. V., Smith, G. B., Vagov, A. V., "Polarization and resonant absorption of intersecting cylinders and spheres," Journal of Applied Physics, Vol. 76, No. 8, 1994, pp. 4827-4835.

23. Mansfield, M. L., Douglas, J. F., Garboczi, E. J., "Intrinsic viscosity and the electrical polarizability of arbitrarily shaped objects," Physics Review E. Vol. 64, No. 061401, 2001. (10 pages).

24. Babuk, V. A., Budnyj, N. L., "Modelirovanie formirovanija oksida pri gorenii metalla aglomeratov," Himicheskaja fizika i mezoskopija, Vol. 17, No. 1, 2015, pp. 39-50.

25. Babuk, V.A., Nizjaev, A.A. K voprosu o formirovanii «polyh» aglomeratov pri gorenii smesevyh tverdyh topliv," Himicheskaja fizika i mezoskopija, Vol. 18, No. 1, 2016, pp. 5-1.

26. Tenenev, V. A., Rusjak, I. G., Gorohov, M. M., "Chislennoe issledovanie gorenija chastic aljuminija v dvuhfaznom potoke," Matematicheskoe modelirovanie, Vol. 9, No. 5, 1997, pp. 87-96.

27. Gremjachkin, V. M., "Teoreticheskie modeli gorenija metallicheskih chastic," Fizika ajerodispersnyh system, No. 31, 1987, pp. 41-52.

28. Strongin, M. P., Koshelev, K. B., "Matematicheskoe modelirovanie nestacionarnyh jeffektov pri obtekanii odinochnoj gorjashhej chasticy," Fizika gorenija i vzryva, Vol. 29, No. 3, 1993, pp. 120-123.

29. French, A. D., Panelli, M., Lorenzo, G. D., Schettino, A., "Combustion instability and pressure oscillation numerical simulation in a solid rocket motor," 53rd AIAA/SAE/ASEE Joint Propulsion Conference, AIAA Paper 2017-4952, July 2017.

30. Volkov, K. N., "Combustion of single aluminium droplet in two-phase flow," Heterogeneous Combustion. Nova Science, 2010, pp. 191-260. 
31. King, M. K., "Aluminum combustion in solid rocket motor environment," Proceedings of the Combustion Institute, Vol. 32, No. 2, 2009, pp. 2107-2114.

32. Yang, J., Yoon, W., "Modeling of aluminum particle combustion with emphasis on the oxide effects and variable transport properties," Journal of Mechanical Science and Technology, Vol. 24, No. 4, 2010, pp. 909-921

33. Glorian, J., Gallier, S., Catoire, L., "On the role of heterogeneous reactions in aluminum combustion," Combustion and Flame, Vol. 168, 2016, pp. 378-392.

34. Muller, M., Davidenko, D., Giovangigli, V., "Computational study of aluminum droplet combustion in different atmospheres," Proceedings of the 7th European Conference for Aeronautics and Space Sciences (EUCASS), July 2017, Milan, Italy. 17 p.

35. Volkov, K. N., Emel'janov, V. N., Teterina, I. V., Jakovchuk, M. S., "Gazovye techenija v soplah jenergoustanovok (Gas flows in power plant nozzles),". M.: Fizmatlit, 2016. 336 c.

36. Volkov, K. N., "Diskretizacija uravnenij Nav'e-Stoksa na nestrukturirovannoj setke pri pomoshhi metoda kontrol'nogo ob\#ema i raznostnyh shem povyshennoj razreshajushhej sposobnosti," Zhurnal vychislitel'noj matematiki i matematicheskoj fiziki, Vol. 48, No. 7, 2008, pp. 1250-1273.

37. Volkov, K. N., "Mnogosetochnye tehnologii dlja reshenija zadach gazovoj dinamiki na nestrukturirovannyh setkah," Zhurnal vychislitel'noj matematiki i matematicheskoj fiziki, Vol. 50, No. 11, 2010, pp. 1938-1952.

38. Volkov, K. N., "Blochnoe predobuslovlivanie uravnenij Jejlera i Nav'e-Stoksa pri modelirovanii nizkoskorostnyh techenij na nestrukturirovannyh setkah," Zhurnal vychislitel'noj matematiki $i$ matematicheskoj fiziki, Vol. 49, No. 10, 2009, pp. 1868-1884. 